\section{Decrease in size of secondary neck vessels and cerebral aque- duct enlargement in multiple sclerosis: A 5-year longitudinal MRI study}

\author{
Dejan Jakimovski, ${ }^{1}$ \\ Maria Marcella Laganà, ${ }^{2}$ \\ Matthew Topolski, ${ }^{1}$ Kana Kimura, ${ }^{1}$ \\ Virja Pandya, ${ }^{1}$ Niels Bergsland, ${ }^{1}$ \\ Bianca Weinstock-Guttman, ${ }^{3}$ \\ Robert Zivadinov ${ }^{1,4}$
}

${ }^{1}$ Buffalo Neuroimaging Analysis Center (BNAC), Department of Neurology, Jacobs School of Medicine and

Biomedical Sciences, University at

Buffalo, State University of New York,

Buffalo, NY, USA; ' IRCCS, Fondazione Don Carlo Gnocchi ONLUS, Milano, Italy; ${ }^{3}$ Jacobs Comprehensive MS Treatment and Research Center, Department of Neurology, Jacobs School of Medicine and Biomedical Sciences, University at Buffalo, State University of New York, NY, USA; ${ }^{4}$ Center for Biomedical Imaging at Clinical Translational Science Institute, University at Buffalo, State University of New York, Buffalo, NY, USA

\section{Background}

Studies have previously shown changes within the cerebrospinal fluid (CSF) dynamics, extracranial vascular arterial, and venous vessels in persons with multiple sclerosis (PwMS) when compared to healthy controls (HCs). ${ }^{1-3}$

\section{Objectives}

To determine the change in CSF dynamics and in number and size of secondary neck vessels in PwMS and HCs over 5-year follow-up period.

\section{Methods}

Both at baseline and follow-up, 83 PwMS and $25 \mathrm{HCs}$ underwent magnetic resonance angiography (MRA) imaging whereas, a subset of 40 PwMS and 20 HCs underwent also longitudinal phase contrast cine imaging. The number and cross-sectional area (CSA) of all secondary neck vessels (excluding the common/internal carotid, vertebral artery, and internal jugular vein) measured at levels from C2-T1 were determined by semi-automated edge detection/contouring software. Measures of CSF Aqueduct of Sylvius (AoS) velocity, average AoS CSA, and systolic and diastolic peaks of velocity and flow rate were determined using Segment version 2.0 (Medvisio, Lund, Sweden, http://segment.heiberg.se). The longitudinal change in CSF and vascular measures was analyzed by non-parametric Wilcoxon repeated measure. For each substudy, Benjamini-Hochberg procedure adjusted for false discovery rate (FDR).

\section{Results}

Over 5 years, PwMS demonstrated consistent longitudinal decrease in both the number of secondary neck vessels (Zchange between -3.3 and $-5.4, \mathrm{q}=0.001)$ and their CSA (Z-change between -2.9 and $-5.2, \mathrm{q}=0.004)$. On the contrary, the HCs did not demonstrate significant longitudinal change in secondary neck vessels over the follow-up period. The subset of PwMS had average AoS CSA increase from $3.1 \mathrm{~mm}^{2}$ to $3.7 \mathrm{~mm}^{2}, \mathrm{q}=0.001$, and increase in diastolic peak flow rate from $7.8 \mathrm{~mL} / \mathrm{min}$ to 9.3 $\mathrm{mL} / \mathrm{min}, \mathrm{P}=0.023$. When compared to $\mathrm{HCs}$, PwMS had greater average AoS CSA (2.6 $\mathrm{mm}^{2}$ vs $\left.3.6 \mathrm{~mm}^{2}, \mathrm{P}=0.045\right)$. The increase in AoS CSA was driven by the progressive MS phenotype $\left(2.6 \mathrm{~mm}^{2} v 3.3 \mathrm{~mm}^{2} v 3.9\right.$ $\mathrm{mm}^{2}$; for HCs, relapsing-remitting MS, and progressive MS, respectively). The change in AoS CSA was not associated with the change in secondary vessels, but with global ventricular CSF expansion rate $\left(r_{\mathrm{s}}=0.457\right.$, $\mathrm{P}=0.025$ ).
Correspondence: Dejan Jakimovski, Buffalo Neuroimaging Analysis Center (BNAC), Department of Neurology, Jacobs School of Medicine and Biomedical Sciences, University at Buffalo, State University of New York, Buffalo, NY, USA.

E-mail: djakimovski@bnac.net

Key words: Neurovascular diseases; meeting.

Conference presentation: $9^{\text {th }}$ Annual Meeting of the International Society of Neurovascular Disease (ISNVD), May $30^{\text {th }}-31^{\text {st }} ， 2019$, Ferrara, Italy.

Award: Young Investigator Award Session First prize.

This work is licensed under a Creative Commons Attribution 4.0 License (by-nc 4.0).

(C) Copyright: the Author(s), 2019

Licensee PAGEPress, Italy

Veins and Lymphatics 2019; 8:8440

doi:10.4081/vl.2019.8440

\section{Conclusions}

PwMS demonstrate significant mid-term decrease in the number and the size of the secondary neck vessels. The significant AoS CSA enlargement may reflect local atrophy of the surrounding brain structures. The clinical relevance of these findings and its effect on intracranial fluid dynamics are currently unknown.

\section{References}

1. Jakimovski D, Marr K, Mancini M, et al. Global and regional brain atrophy is associated with low or retrograde facial vein flow in multiple sclerosis. Veins and Lymphatics 2017;6:6976.

2. Beggs C. Cerebral venous outflow and cerebrospinal fluid dynamics. Veins and Lymphatics 2014;3:1867.

3. Jakimovski D, Topolski M, Genovese $\mathrm{AV}$, et al. Vascular aspects of multiple sclerosis: emphasis on perfusion and cardiovascular comorbidities. Expert Rev Neurother 2019;19:445-58. 